Article

\title{
Evidence of Slow Magnetic Relaxation in $\mathrm{Co}(\mathrm{AcO})_{2}(\mathrm{py})_{2}\left(\mathrm{H}_{2} \mathrm{O}\right)_{2}$
}

\author{
James P. S. Walsh ${ }^{1,2}$, Graeme Bowling ${ }^{3}$, Ana-Maria Ariciu ${ }^{1}$, Nur F. M. Jailani ${ }^{1}$, \\ Nicholas F. Chilton ${ }^{1, *}$, Paul G. Waddell ${ }^{3}$, David Collison ${ }^{1}$, Floriana Tuna ${ }^{1, *}$ and Lee J. Higham ${ }^{3, *}$ \\ 1 School of Chemistry, University of Manchester, Oxford Road, Manchester M13 9PL, UK; \\ james.walsh@northwestern.edu (J.P.S.W.); ana-maria.ariciu@manchester.ac.uk (A.-M.A.); \\ nurfaizah.mohdjailani@postgrad.manchester.ac.uk (N.F.M.J.); david.collison@manchester.ac.uk (D.C.) \\ 2 Department of Chemistry, Northwestern University, Evanston, IL 60208, USA \\ 3 School of Chemistry, Bedson Building, Newcastle University, Newcastle upon Tyne NE1 7RU, UK; \\ g.bowling@newcastle.ac.uk (G.B.); paul.waddell@ncl.ac.uk (P.G.W.) \\ * Correspondence: nicholas.chilton@manchester.ac.uk (N.F.C.); floriana.tuna@manchester.ac.uk (F.T.); \\ lee.higham@newcastle.ac.uk (L.J.H.); Tel.: +4416-1275-4584 (N.F.C.); +4416-1275-1005 (F.T.); \\ +4419-1208-5542 (L.J.H.)
}

Academic Editor: Marius Andruh

Received: 1 March 2016; Accepted: 13 April 2016; Published: 20 April 2016

\begin{abstract}
The monometallic pseudo-octahedral complex, $\left[\mathrm{Co}\left(\mathrm{H}_{2} \mathrm{O}\right)_{2}\left(\mathrm{CH}_{3} \mathrm{COO}\right)_{2}\left(\mathrm{C}_{5} \mathrm{H}_{5} \mathrm{~N}\right)_{2}\right]$, is shown to exhibit slow magnetic relaxation under an applied field of $1500 \mathrm{Oe}$. The compound is examined by a combination of experimental and computational techniques in order to elucidate the nature of its electronic structure and slow magnetic relaxation. We demonstrate that any sensible model of the electronic structure must include a proper treatment of the first-order orbital angular momentum, and we find that the slow magnetic relaxation can be well described by a two-phonon Raman process dominating at high temperature, with a temperature independent quantum tunnelling pathway being most efficient at low temperature.
\end{abstract}

Keywords: single-ion magnet; slow relaxation; magnetic anisotropy; zero-field splitting; cobalt

\section{Introduction}

The study of paramagnetic molecules exhibiting a measurably slow relaxation of their magnetisation is a field that has been growing since the observation of so-called single-molecule magnet (SMM) behaviour in a dodecametallic mixed-valence manganese cluster just over two decades ago [1]. The vast majority of the work that has been reported since then has focused on the development of new chemical systems that exhibit the same property above liquid helium temperatures. Although initial work in this direction involved transition metal clusters, the discovery of SMM behaviour in monometallic complexes-firstly in lanthanides [2,3], but more recently in transition metals [4-6] —has caused a shift away from cluster compounds due to the comparative ease with which monometallic systems can be chemically tuned.

In many ways, the complexity of the magnetic properties in monometallic systems is greatly reduced compared to polymetallic clusters on account of the lack of magnetic exchange interactions. However, a complete understanding of the intrinsic single-ion properties of compounds that exhibit slow relaxation remains elusive [7]. The main reason for this is the complexity that arises from spin-orbit coupling, one of the necessary prerequisites for slow magnetic relaxation.

For a compound to exhibit slow magnetic relaxation, it must have a doubly degenerate ground state comprised of states with opposite magnetic projections separated by an energy barrier of sufficient height to inhibit inversion after the removal of an external magnetic field. If the barrier is large with 
respect to temperature then the system will remain in the given ground state indefinitely at zero field. The temperature above which the magnetic blocking is no longer effective is defined as the blocking temperature, $T_{\mathrm{B}}$. The value of $T_{\mathrm{B}}$ is tenuously related to the height of the empirically determined effective energy barrier, $U_{\text {eff, }}$ which is usually lower than the actual separation between the highest and lowest energy states, $\Delta$, due to competing relaxation pathways.

For ions which have first-order orbital angular momentum, the total angular momentum, $J$, is often subject to large magnetic anisotropy due to the splitting of the orbital states; this anisotropy is then projected onto the ground J multiplet by spin-orbit coupling. Alternatively, if the ion's ground state is spin-only (i.e., an orbital singlet), then the magnetic anisotropy arises instead from mixing of orbitally-degenerate excited states into the ground state, via spin orbit coupling. This weaker effect is often modelled as a zero-field splitting, using the axial and rhombic zero-field splitting (ZFS) terms, $D$ and $E$, respectively [8]. For polymetallic systems, $\Delta$ is influenced not only by the intrinsic magnetic anisotropy of the constituent ions, but also by the total spin of the ground state, which is in turn dictated by exchange interactions. In monometallic systems however, no intramolecular exchange interactions exist, and consequently $\Delta$ is defined solely by the single site magnetic anisotropy.

The foregoing discussion makes the implicit assumption that slow relaxation must be in some part due to an intrinsic barrier between the ground states-a so-called double well configuration that places the largest $m_{S}$ or $m_{J}$ states lowest in energy with the other states forming a barrier between them; in other words, it implicitly assumes that a negative axial ZFS parameter gives rise to a real barrier to relaxation. For this barrier to be the source of slow relaxation further assumes that an Orbach relaxation process is dominant. However, it is well known that when $\Delta$ is very large, other thermally activated processes, such as Raman processes, become important $[3,9,10]$.

The double well model holds up in the case of monometallic cobalt(II) SMMs with negative $D$ values [9,11-19], but the observation of similar behaviour in monometallic cobalt(II) complexes with positive $D$ values [20-22] suggests that the picture is more complex. Indeed, slow relaxation in positive- $D$ compounds is a phenomenon that is not yet fully understood-although attempts have been made to explain it $[23,24]$. The apparent unimportance of $D$ as a design parameter in general has also been noted by others [25].

A factor that is sometimes overlooked is the complexity that arises in systems containing first-order orbital angular momentum, and more specifically the fact that the electronic structure of such systems cannot be modelled by simple ZFS models associated with "easy-axis" and "easy-plane" magnetisation. Slow-relaxing systems whose magnetic behaviour is incorrectly modelled in this way may inadvertently obfuscate our understanding of the true source of their slow relaxation, especially when the anisotropy is clearly rhombic in nature rather than axial.

Herein, we report slow magnetic relaxation behaviour in the monometallic pseudo-octahedral cobalt(II) compound, $\left[\mathrm{Co}\left(\mathrm{H}_{2} \mathrm{O}\right)_{2}\left(\mathrm{CH}_{3} \mathrm{COO}\right)_{2}\left(\mathrm{C}_{5} \mathrm{H}_{5} \mathrm{~N}\right)_{2}\right]$, and further we examine the nature of the relaxation barrier in the context of a model that accounts for the first-order orbital angular momentum.

\section{Results and Discussion}

The monometallic cobalt(II) compound $\left[\mathrm{Co}\left(\mathrm{H}_{2} \mathrm{O}\right)_{2}\left(\mathrm{CH}_{3} \mathrm{COO}\right)_{2}\left(\mathrm{C}_{5} \mathrm{H}_{5} \mathrm{~N}\right)_{2}\right](\mathbf{1})$ was synthesized as previously reported [26]. Its crystal structure consists of a cobalt(II) ion coordinated by two pyridines, two acetates, and two water molecules, with each of the ligands occupying coordination positions trans to each other (Figure 1). It crystallises in the Pbca space group with an inversion centre on the cobalt atom and therefore with half of the molecule in the asymmetric unit. Relevant bond lengths and angles are summarised in Table 1. The coordination environment around the cobalt centre is pseudo-octahedral, with bond lengths and angles falling within the narrow range of 2.08-2.16 $\AA$ and $88.9^{\circ}-92.7^{\circ}$, respectively. The closest intermolecular Co ‥ Co separation is $7.72 \AA$. 

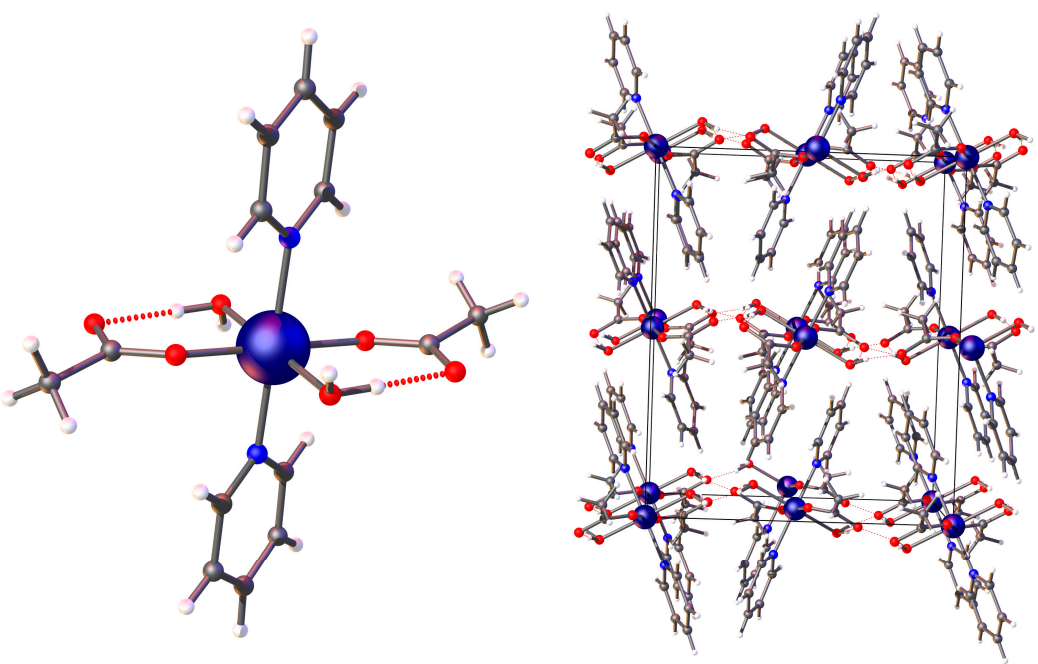

Figure 1. Crystal structure of compound 1 and a packing diagram viewing almost along the $b$-axis.

Table 1. Selected bond lengths and angles for compound $\mathbf{1 .}$

\begin{tabular}{cccc}
\hline \multicolumn{2}{c}{ Bond Length (̊̊) } & \multicolumn{2}{c}{ Angle $\left({ }^{\circ}\right)$} \\
\hline Co1-O1 & $2.076(2)$ & O1-Co1-O2 & $88.78(8)$ \\
Co1-O3 & $2.121(2)$ & O1-Co1-N1 & $90.54(10)$ \\
Co1-N1 & $2.159(3)$ & O2-Co1-N1 & $92.69(10)$ \\
\hline
\end{tabular}

A plot of $\chi_{\mathrm{M}} T(T)$ for compound $\mathbf{1}$ is shown in Figure 2. The form of the trace is typical for a monometallic $\mathrm{Co}$ (II) species influenced by an orbital contribution to the magnetic moment. The room temperature $\chi_{\mathrm{M}} T$ value is $3.04 \mathrm{~cm}^{3} \mathrm{~K} \mathrm{~mol}^{-1}$, which is significantly higher than the calculated spin-only value for an $S=3 / 2$ ion with $g=2\left(1.875 \mathrm{~cm}^{3} \mathrm{~K} \mathrm{~mol}^{-1}\right)$, and is within the range expected for ochahedral Co(II) complexes [8]. With decreasing temperature, $\chi_{\mathrm{M}}^{T}$ falls to $1.44 \mathrm{~cm}^{3} \mathrm{~K} \mathrm{~mol}^{-1}$ at 2 K. Field-dependent magnetisation measurements carried out at low temperatures (Figure 2, inset) exhibit near saturation at $7 \mathrm{~T}$-indicative of a well-isolated ground state-to a value of around $2.3 N \mu_{B}$. Reduced magnetisation plots exhibit only minor separations over the full field range and up to $20 \mathrm{~K}$ (Figure S1), which is further evidence of an isolated ground state.

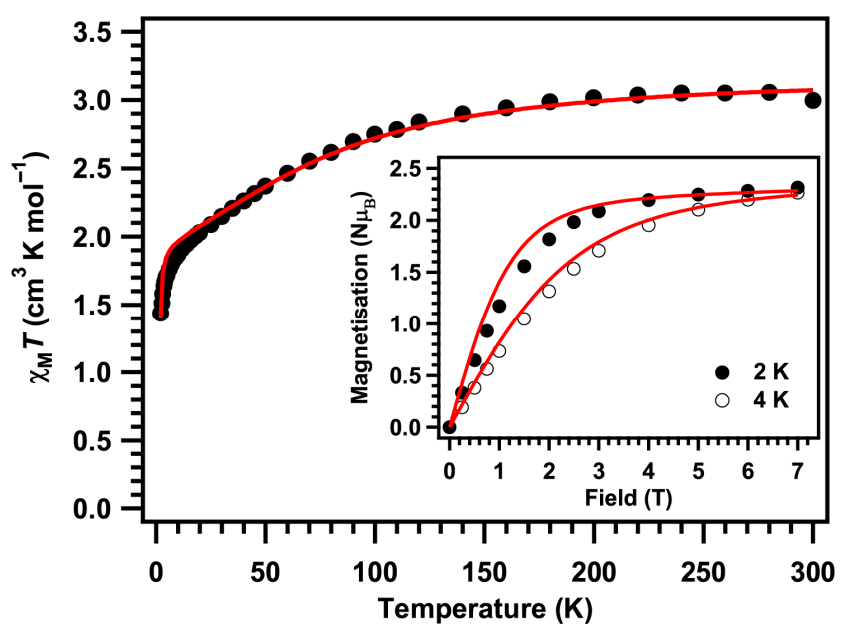

Figure 2. Plots of $\chi_{M} T(T)$ measured under a static field of $0.1 \mathrm{~T}$ (main) and field-dependent magnetisation at 2 and $4 \mathrm{~K}$ (inset) for compound 1. Red traces are simulations using the $\mathrm{T}, \mathrm{P}$ isomorphism model described in the main text. 
In the absence of any close contacts between individual molecules, the downward turn in the trace of $\chi M T(T)$ upon reducing temperature must be caused primarily by depopulation of the spin-orbit split crystal field states of the octahedral cobalt(II) centre. We further probed the ground state by carrying out low temperature $(<20 \mathrm{~K})$ electron paramagnetic resonance (EPR) spectroscopy on a powder sample (Figure S2). The spectrum exhibits features over a field range consistent with a rhombic ground doublet. Given that the energy gap to the first excited state is so large due to the strong spin-orbit coupling, we can treat the ground Kramers doublet as an effective $\widetilde{S}=1 / 2$ state according to the following Hamiltonian:

$$
\hat{H}=\mu_{B}\left(g_{x} \widetilde{S}_{x} H_{x}+g_{y} \widetilde{S}_{y} H_{y}+g_{z} \widetilde{S}_{z} H_{z}\right)
$$

In such a model, the anisotropy of the ground state is modelled well with effective $g$-values, $g_{x}=2.17, g_{y}=3.45, g_{z}=6.65$, which is in very good agreement with the values calculated for this ground state by ab initio methods (see below). A number of small features appear in between these dominant absorptions whose origin is not obvious. We cannot rule out the possibility of small intermolecular exchange interactions, which could be weak enough to be undetectable by susceptibility measurements, yet sufficient to cause additional transitions in the EPR spectrum over the measured field ranges (i.e., on the order of a few wavenumbers). The inherent complexity of such an exchange model would warrant a more extensive dataset than currently available.

To support our interpretation of the magnetic and spectroscopic data, ab initio calculations of the complete active space self-consistent field (CASSCF) variety were performed using MOLCAS 8.0 [27] (see Supplementary Materials for details). The results show that the ground orbital triplet of the high-spin octahedral cobalt(II) site is split owing to low-symmetry distortions in the solid state, and that this splitting is weaker than the spin-orbit coupling. The ground state Kramers doublet is predicted to be strongly anisotropic with $\widetilde{S}=1 / 2$ effective $g$-values, $g_{x}=2.39, g_{y}=3.28, g_{z}=7.15$, which compare extremely well with the experimental spectroscopic data. These $g$-values are more-or-less associated with the different ligand pairs, all of which are trans- to each other; the principal direction for the smallest $g$-value, $g_{x}$, is along the water-water axis, for the intermediate value, $g_{y}$, it is along the pyridine-pyridine axis, and for the largest value, $g_{z}$, it is along the acetate-acetate axis (Figure 3). The first excited doublet is predicted to lie at around $264 \mathrm{~cm}^{-1}$ above the ground state, supporting the measurement of a well isolated ground state by magnetometry.

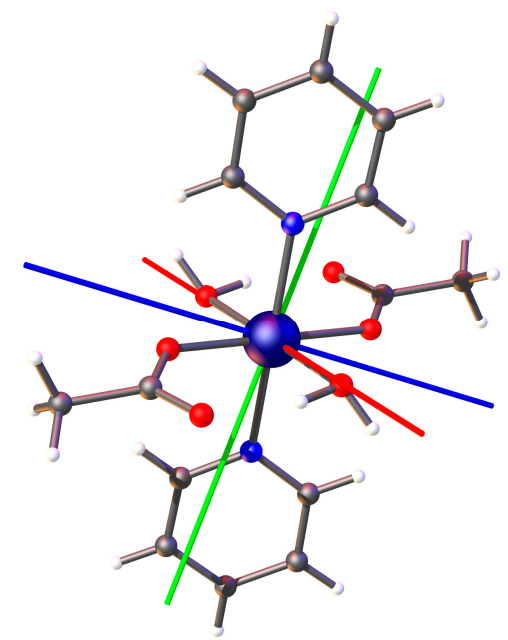

Figure 3. Orientation of the principal axes for the $\widetilde{S}=1 / 2$ effective $g$-values of the ground Kramers doublet for 1 . Red $=$ hard axis, $g_{x}$; green $=$ intermediate axis, $g_{y}$; blue $=$ easy axis, $g_{z}$.

A simple approach to account for the first-order orbital angular momentum associated with the ${ }^{4} \mathrm{~T}_{1 \mathrm{~g}}$ ground state is to employ the $\mathrm{T}$, $\mathrm{P}$ isomorphism with an effective orbital moment of $\widetilde{L}=1$, where the coefficient of proportionality is $-3 / 2$ [28]. In this model, we fix the spin-orbit coupling constant 
for cobalt(II) to $\lambda=-170 \mathrm{~cm}^{-1}$ and we do not employ an orbital reduction factor [29], in order to avoid overparameterisation. Using this model, the free-ion electronic structure of $\mathrm{Co}$ (II) defines three spin-orbit states of $J=1 / 2,3 / 2$ and $5 / 2$, given in order of increasing energy (Table S1). The low symmetry distortions of the octahedral coordination environment split these $J$ states and mix them together, altering the $g$-value of the $J=1 / 2$ ground state from $g_{i s o}=4.33$ (Table S2). We model this using effective crystal-field splitting parameters acting on the effective $\widetilde{L}=1$ orbital moment, and these are used as fitting parameters to reproduce the EPR results using the PHI code [30]. In order to reproduce the pattern of $g_{x} \approx g_{y}<g_{z}$ in the ground state, the $B_{2}^{0}$ parameter must be negative, and we find excellent agreement with the EPR $g$-values using $B_{2}^{0}=-124 \mathrm{~cm}^{-1}$ and $\left|B_{2}^{2}\right|=109 \mathrm{~cm}^{-1}$ with the Hamiltonian given in Equation (2). This parameterisation also provides extremely good agreement to the experimental magnetic data (see above) and EPR $g$-values (Table S3).

$$
\hat{H}=-\frac{3}{2} \lambda \hat{L} \cdot \hat{S}+\frac{9}{4}\left(B_{2}^{0}\left(3 \hat{L}_{z}{ }^{2}-\hat{L}^{2}\right)+\frac{B_{2}^{2}}{2}\left(\hat{L}_{+}{ }^{2}+\hat{L}_{-}{ }^{2}\right)\right)+\mu_{B}\left(-\frac{3}{2} \hat{L}+2 \hat{S}\right) \cdot H
$$

Ac susceptibility measurements were carried out on powder samples of compound 1 . Figure 4a shows the frequency dependence of the out-of-phase component of the ac susceptibility at $1.8 \mathrm{~K}$ under applied fields of strength between 0 and 5000 Oe. A peak in the out-of-phase (imaginary) susceptibility manifested under applied external fields, and reached a maximum shift to low frequencies for $1500 \mathrm{Oe}$, which we consider as the optimal field under which to observe slow magnetic relaxation. A plot of the magnitude of the relaxation time as a function of the applied magnetic field is shown in Figure $4 \mathrm{~b}$.

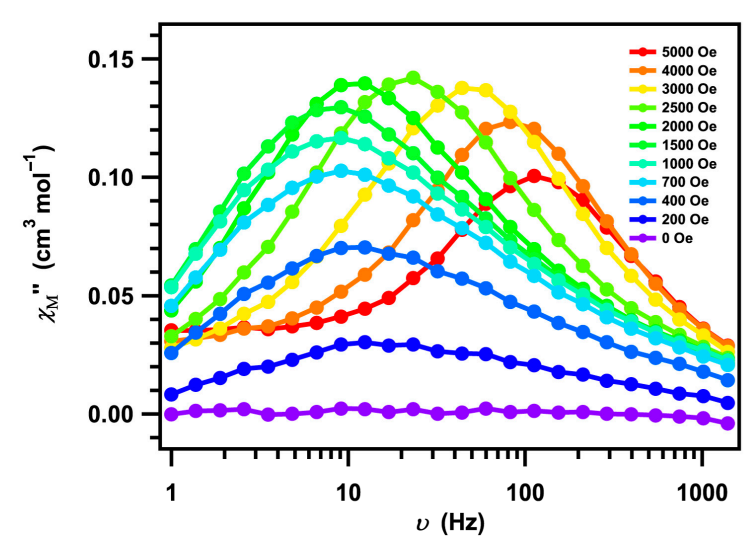

(a)

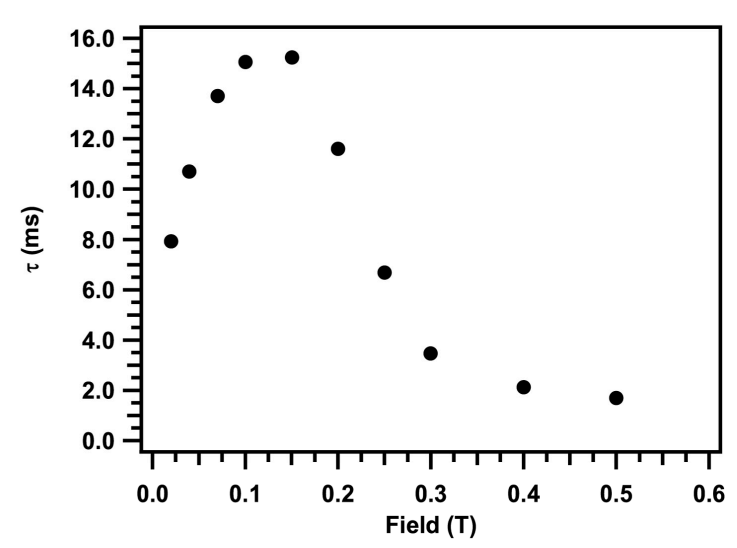

(b)

Figure 4. (a) Frequency dependence of the imaginary ac susceptibility of $\mathbf{1}$ measured at $1.8 \mathrm{~K}$ and under several applied fields. The lines are guides for the eye; (b) Field dependence of the relaxation time at $1.8 \mathrm{~K}$.

Further ac susceptibility data were collected at this field over a range of temperatures (1.8-10 K) and oscillation frequencies (1-1400 Hz) (Figures 5 and 6). At fixed temperatures between 1.8 and $7.2 \mathrm{~K}$, semicircle Cole-Cole plots were generated and fit using the generalised Debye model assuming a single relaxation pathway to extract the magnetisation relaxation time $(\tau)$ at each temperature (Figure 7$)$. The $\alpha$ parameter, which is a measure of the distribution of relaxation times, varies over the range $0.41-0.03$ for measurements at fixed temperatures between 1.8 and $7.2 \mathrm{~K}$. As the fits in the $1.8-3.2 \mathrm{~K}$ temperature regime fail to reproduce the peak in the out-of-phase susceptibility, the relaxation times extracted using the Debye fits are not reliable and therefore for the five lowest temperatures (1.8-3.2 K) we extracted the relaxation times directly from the peak position of the out-of-phase susceptibility. These were used to build the Arrhenius plot given in the inset of Figure 7. 


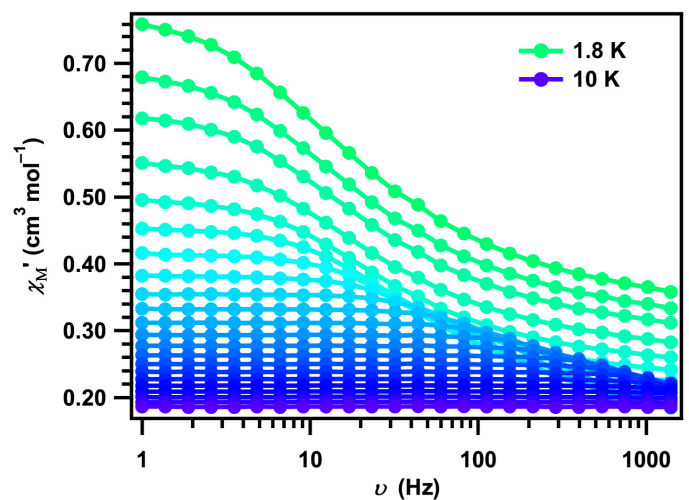

(a)

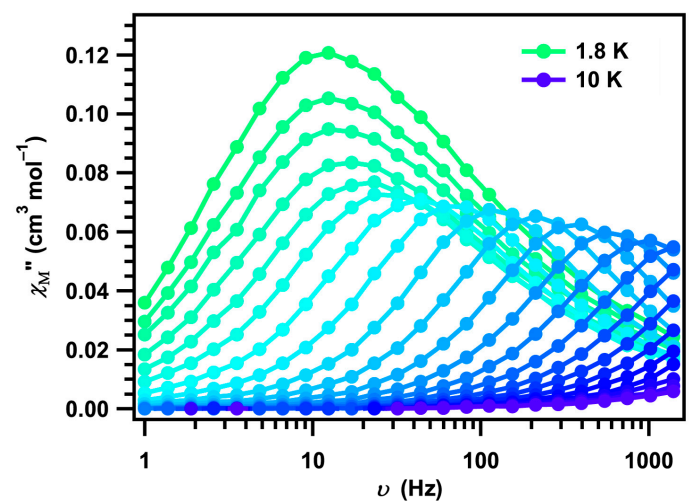

(b)

Figure 5. Frequency dependence of the (a) real and (b) imaginary ac susceptibilities of $\mathbf{1}$ measured under a static field of 1500 Oe and temperatures $1.8-10 \mathrm{~K}$. Solid lines are guides for the eye.

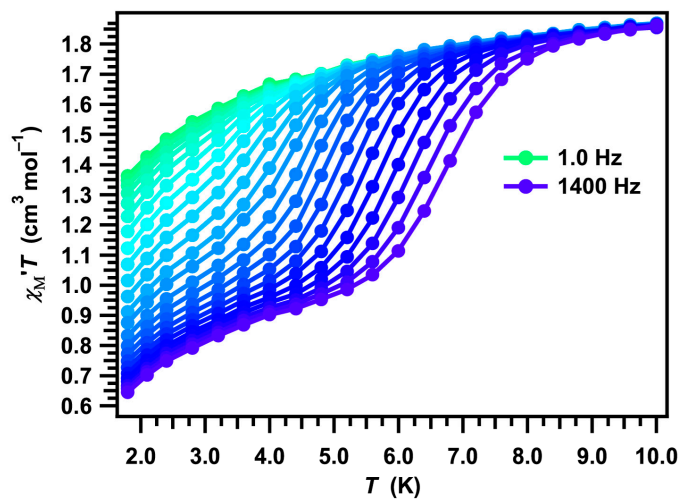

(a)

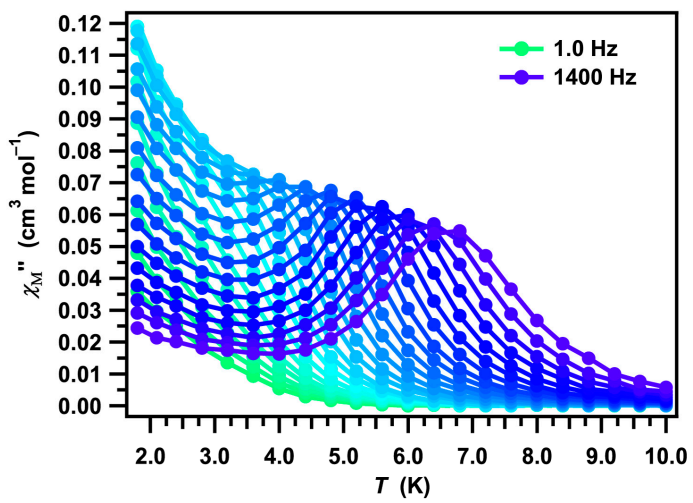

(b)

Figure 6. Temperature dependence of (a) $\chi^{\prime}{ }_{M} T$ and (b) $\chi^{\prime \prime}{ }_{M}$ for 1 measured under a static field of $1500 \mathrm{Oe}$ and an ac field of 1.55 Oe oscillating at frequencies from 1 to $1400 \mathrm{~Hz}$.

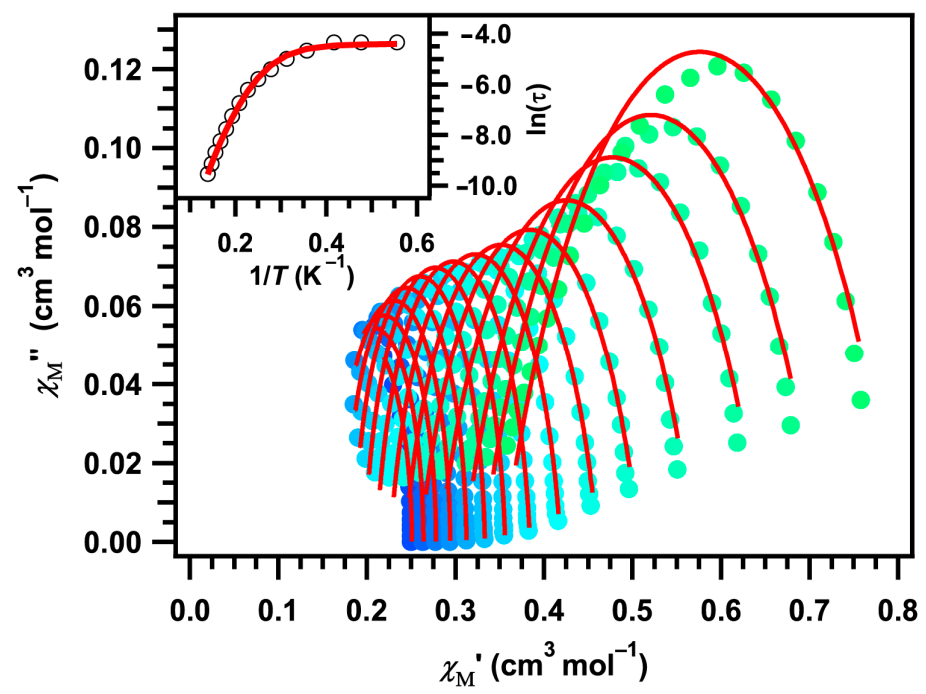

Figure 7. Cole-Cole plot of the measured ac susceptibility data for 1 showing fits obtained using the generalised Debye model (red traces). Inset graph shows the plot of $\ln (\tau)$ against $1 / T$, and a fit to Equation (4) with the parameters given in the text (red line). 
The relaxation times were modelled in an attempt to determine the mechanism(s) of magnetic relaxation. The low temperature regime clearly shows temperature-independent behaviour, which is the hallmark of quantum tunnelling of the magnetization (QTM). The high temperature region can be fit equally well with two different models, one assuming an Orbach relaxation mechanism and the other assuming a Raman relaxation mechanism. The former, using Equation (3), provides the values $U_{\text {eff }}=25 \mathrm{~cm}^{-1}(36 \mathrm{~K}), \tau_{0}=6.7 \times 10^{-7} \mathrm{~s}$, and $\tau_{\mathrm{QTM}}=1.1 \times 10^{-2} \mathrm{~s}$ (Figure S3), while the latter, using Equation (4), provides the values $C=1.4 \times 10^{-2} \mathrm{~s}^{-1} \mathrm{~K}^{-7}, n=7$, and $\tau_{\mathrm{QTM}}=1.2 \times 10^{-2} \mathrm{~s}$ (Figure 7, inset).

$$
\begin{gathered}
\tau^{-1}=\tau_{Q T M}{ }^{-1}+\tau_{0}{ }^{-1} e^{-U_{e f f} / k_{B} T} \\
\tau^{-1}=\tau_{Q T M^{-1}}+C T^{n}
\end{gathered}
$$

While both models provide very good agreement with the experimental data, the use of the Orbach model implies that a real state exists at an energy separation of $25 \mathrm{~cm}^{-1}$ above the ground state to provide the intermediate state in the magnetic relaxation pathway. From our magnetometry measurements and CASSCF calculations, the first excited state is known to be well separated from the ground state and is predicted to lie at $\sim 264 \mathrm{~cm}^{-1}$. On this basis, we propose that the Raman mechanism is dominant in the high temperature regime. Indeed, the $n$ parameter of 7 is entirely commensurate with a two-phonon Raman process for a Kramers ion such as $\mathrm{Co}(\mathrm{II})$, and the $C$ value is in the expected range of $10^{-5}$ to $10^{-1}$ [31]. Two-phonon Raman relaxation was reported for single-ion magnets based on both d- [9] and f-block [3,10] metals.

Compared to cases where the orbital angular momentum is quenched and $S=3 / 2$ is an appropriate model for the electronic structure, pseudo-octahedral Co(II) requires a more detailed model, an example of which has been employed here. In such situations, discussion of "easy-axis" vs. "easy-plane" anisotropy corresponding to negative and positive $D$, respectively, is entirely inappropriate. Indeed, as we have demonstrated here, we can often experimentally verify that we instead have a significantly rhombic electronic structure where $g_{x} \neq g_{y} \neq g_{z}$. The fact that the principal directions of the three $g$-values are nearly coincident with the Co - ligand donor atom directions strongly suggests that the different electronic structures of the ligands and their coordination modes are directly responsible for the crystal field distortions that lead to the rhombic $g$-values. Indeed, the short coordination bonds to the acetate oxygen atoms (2.0762 (18) $\AA$ ) coupled with the negative charge of these anions is likely responsible for dictating the easy axis. It is difficult to ascertain the origin of the significant rhombicity, but it is likely due to the difference between the $\sigma$-type lone pair of pyridine compared to the $\sigma$ - and $\pi$-type lone pair of water.

In conclusion, compound $\mathbf{1}$ is an excellent example of a pseudo-octahedral Co(II) complex with a rhombic electronic structure whose magnetic behaviour can only be sensibly modelled by considering the effects of the first-order orbital angular momentum. We found a large discrepancy between the calculated $U_{\text {eff }}$ value assuming an Orbach relaxation mechanism and the value of $\Delta$ deduced from fits to the dc magnetic data, suggesting that there is no such effective barrier, $U_{\text {eff }}$, in this case. The most likely slow relaxation pathway in $\mathbf{1}$ is a two-phonon Raman process dominating at high temperature, with a temperature-independent quantum tunnelling pathway being most efficient at low temperature. This quantum tunnelling relaxation is likely to occur via dipolar interactions that occur in the solid-state [10], and/or intramolecular hyperfine coupling interactions [32]. Further studies are necessary to clarify this aspect.

\section{Materials and Methods}

The synthesis of 1 was carried out according to a known literature procedure [26]. All magnetic measurements were performed using a Quantum Design MPMS-XL SQUID magnetometer (Quantum Design, San Diego, TX, USA) equipped with a 7 T magnet. The sample was ground to a powder, loaded into a gelatin capsule, and fixed in eicosane (Sigma-Aldrich, Dorset, UK). Sample mass $=9.2 \mathrm{mg}$, eicosane mass $=6.0 \mathrm{mg}$. Temperature-dependent susceptibility measurements were carried out under 
a static field of $0.1 \mathrm{~T}$ and in the temperature range 2-300 K. Field dependent/reduced magnetisation measurements were carried out under magnetic fields between 0 and $7 \mathrm{~T}$ and over the temperature range of $1.8-20 \mathrm{~K}$. Ac susceptibility was measured using a $1.55 \mathrm{Oe}$ ac field oscillating at frequencies between 0.1 and $1400 \mathrm{~Hz}$ and over the temperature range of 1.8-12.0 K. The optimum static field for ac (alternating current) measurements (1500 Oe) was determined by measuring the frequency dependence of the out-of-phase component of the susceptibility at $1.8 \mathrm{~K}$ between 0 and 5000 Oe. Diamagnetic corrections were applied using a combination of tabulated Pascal constants for the sample diamagnetism, and a subtraction of the sample holder behaviour measured under comparable sequences. CASSCF calculations were conducted using the X-ray crystallographic geometry with no modifications. The ANO-RCC-VTZP basis set was employed for Co and ANO-RCC-VDZP was used for all other atoms [33,34]. All 10 quartet states and 40 doublet states of the $3 d^{7}$ configuration (CAS 7 in 5) were employed for the orbital optimisation and were mixed by spin-orbit coupling.

Supplementary Materials: The following are available online at www.mdpi.com/2312-7481/2/2/23/s1. X-band EPR, Orbach fit of Arrhenius plot, tabulated details of computational modelling.

Acknowledgments: This work has been supported by EPSRC UK via a Career Acceleration Fellowship award to L.J.H. (EP/G005206/1) and funding of the National EPR Research Facility and Service at the University of Manchester. J.P.S.W. and A.-M.A. acknowledge the NoWNANO Doctoral Training Centre at Manchester and the European Commission MAGnetic Innovation in Catalysis Initial Training Network for doctoral scholarships. We also acknowledge financial support from the School of Chemistry, University of Newcastle for a studentship.

Author Contributions: G.B. synthesized the samples with direction from L.J.H. J.P.S.W., N.F.M.J., and A.-M.A. collected the magnetic and spectroscopic data. J.P.S.W., A.-M.A., and N.F.C. analysed the magnetic and spectroscopic data with direction from F.T. and D.C. N.F.C. performed the theoretical calculations. P.G.W. solved the crystal structure. J.P.S.W., N.F.C., and F.T. wrote the paper, with input from all the co-authors.

Conflicts of Interest: The authors declare no conflict of interest.

\section{Abbreviations}

The following abbreviations are used in this manuscript:

$\begin{array}{ll}\text { SMM } & \text { Single-molecule magnet } \\ \text { ZFS } & \text { Zero-field splitting } \\ \text { EPR } & \text { Electron paramagnetic resonance }\end{array}$

\section{References}

1. Sessoli, R.; Gatteschi, D.; Caneschi, A.; Novak, M.A. Magnetic bistability in a metal-ion cluster. Nature 1993, 365, 141-143. [CrossRef]

2. Ishikawa, N.; Sugita, M.; Ishikawa, T.; Koshihara, S.; Kaizu, Y. Lanthanide double-decker complexes functioning as magnets at the single-molecular level. J. Am. Chem. Soc. 2003, 125, 8694-8695. [CrossRef] [PubMed]

3. Gregson, M.; Chilton, N.F.; Ariciu, A.-M.; Tuna, F.; Crowe, I.F.; Lewis, W.; Blake, A.J.; Collison, D.; McInnes, E.J.L.; Winpenny, R.E.P.; et al. A monometallic lanthanide bis (methanediimide) single molecule magnet with a large energy barrier and complex spin relaxation behavior. Chem. Sci. 2016, 7, 155-165. [CrossRef]

4. Zadrozny, J.M.; Xiao, D.J.; Atanasov, M.; Long, G.J.; Grandjean, F.; Neese, F.; Long, J.R. Magnetic blocking in a linear iron(I) complex. Nat. Chem. 2013, 5, 577-581. [CrossRef] [PubMed]

5. Freedman, D.E.; Harman, W.H.; Harris, T.D.; Long, G.J.; Chang, C.J.; Long, J.R. Slow-relaxation in a high-spin iron(II) complex. J. Am. Chem. Soc. 2010, 132, 1224-1225. [CrossRef] [PubMed]

6. Bar, A.K.; Pichon, C.; Gogoi, N.; Duhayon, C.; Ramasesha, S.; Sutter, J.-P. Single ion behaviour of heptacoordinated Fe(II) complexes: on the importance of supramolecular organization. Chem. Commun. 2015, 51, 3616-3619. [CrossRef] [PubMed]

7. Craig, G.A.; Murrie, M. 3d single ion magnets. Chem. Soc. Rev. 2015, 44, 2135-2147. [CrossRef] [PubMed]

8. Kahn, O. Molecular Magnetism; Wiley-Blackwell: New York, NY, USA, 1993. 
9. Rechkemmer, Y.; Breitgoff, F.D.; van der Meer, M.; Atanasov, M.; Hake, M.; Orlita, M.; Neugetsauer, P.; Neese, F.; Sarkar, B.; van Slageren, J. A four-coordinate cobalt(II) single-ion magnet with coercivity and very high energy barrier. Nat. Commun. 2016, 7. [CrossRef] [PubMed]

10. Titos-Padilla, S.; Ruiz, J.; Herrera, J.M.; Brechin, E.K.; Wernsdorfer, W.; Lloret, F.; Colacio, E. New relativistic ANO basis sets for transition metal atoms. Inorg. Chem. 2013, 52, 9620-9626. [CrossRef] [PubMed]

11. Zadrozny, J.M.; Long, J.R. Slow magnetic relaxation at zero field in the tetrahedral complex $\left[\mathrm{Co}(\mathrm{SPh})_{4}\right]^{2-}$. J. Am. Chem. Soc. 2011, 133, 20732-20734. [CrossRef] [PubMed]

12. Buchholz, A.; Eseola, A.O.; Plass, W. Slow magnetic relaxation in mononuclear tetrahedral cobalt(II) complexes with 2-(1H-imidazol-2-yl)phenol based ligands. Comptes Rendus Chim. 2012, 15, 929-936. [CrossRef]

13. Zhu, Y.-Y.; Cui, C.; Zhang, Y.-Q.; Jia, J.-H.; Guo, X.; Gao, C.; Qian, K.; Jiang, S.-D.; Wang, B.-W.; Wang, Z.-M.; et al. Zero-field slow magnetic relaxation from single $\mathrm{Co}$ (II) ion: a transition metal single-molecule magnet with high anisotropy barrier. Chem. Sci. 2013, 4, 1802-1806. [CrossRef]

14. Zadrozny, J.M.; Telser, J.; Long, J.R. Slow magnetic relaxation in the tertrahedral cobalt(II) complexes $\left[\mathrm{Co}(\mathrm{EPh})_{4}\right]^{2-}(\mathrm{E}=\mathrm{O}, \mathrm{S}, \mathrm{Se})$. Polyhedron 2013, 64, 209-217. [CrossRef]

15. Novikov, V.V.; Pavlov, A.A.; Nelyubina, Y.V.; Boulon, M.E.; Varzatskii, O.A.; Voloschin, Y.Z.; Winpenny, R.E.P. A trigonal prismatic mononuclear cobalt(II) complex showing single-molecule magnet behavior. J. Am. Chem. Soc. 2015, 137, 9792-9795. [CrossRef] [PubMed]

16. Boča, R.; Miklovič, J.; Titiš, J. Simple mononuclear cobalt(II) complex: A single-molecule magnet showing two slow relaxation processes. Inorg. Chem. 2014, 53, 2367-2369. [CrossRef] [PubMed]

17. Saber, M.R.; Dunbar, K.R. Ligands effects on the magnetic anisotropy of tetrahedral cobalt complexes. Chem. Commun. 2014, 50, 12266-12269. [CrossRef] [PubMed]

18. Fataftah, M.S.; Zadrozny, J.M.; Rogers, D.M.; Freedman, D.E. A mononuclear transition metal single-molecule magnet in a nuclear spin-free ligand environment. Inorg. Chem. 2014, 53, 10716-10721. [CrossRef] [PubMed]

19. Cucos, P.; Tuna, F.; Sorace, L.; Matei, I.; Maxim, C.; Shova, S.; Gheorghe, R.; Caneschi, A.; Hillebrand, M.; Andruh, M. Magnetic and luminescent binuclear double-stranded helicates. Inorg. Chem. 2014, 53, 7738-7747. [CrossRef] [PubMed]

20. Zadrozny, J.M.; Liu, J.; Piro, N.A.; Chang, C.J.; Hill, S.; Long, J.R. Slow magnetic relaxation in a pseudotetrahedral cobalt(II) complex with easy-plane anisotropy. Chem. Commun. 2012, 48, 3927-3929. [CrossRef] [PubMed]

21. Huang, W.; Liu, T.; Wu, D.; Cheng, J.; Ouyang, Z.W.; Duan, C. Field-induced slow-relaxation of magnetization in a tetrahedral Co(II) complex with easy plane anisotropy. Dalton Trans. 2013, 42, 15326-15331. [CrossRef] [PubMed]

22. Vaidya, S.; Upadhyay, A.; Singh, S.K.; Langley, S.K.; Walsh, J.P.S.; Murray, K.S.; Rajaraman, G.; Shanmugam, M. A synthetic strategy for switching the single ion anisotropy in tetrahedral cobalt(II) complexes. Chem. Commun. 2014, 51, 3739-3742. [CrossRef] [PubMed]

23. Colacio, E.; Ruiz, J.; Cremades, E.; Krzystek, J.; Carretta, S.; Cano, J.; Guidi, T.; Wernsdorfer, W.; Brechin, E.K. Slow magnetic relaxation in a $\mathrm{Co}^{\mathrm{II}}-\mathrm{Y}^{\mathrm{III}}$ single-ion magnet with positive axial zero-field splitting. Angew. Chem. Int. Ed. 2013, 52, 9130-9134. [CrossRef] [PubMed]

24. Gómez-Coca, S.; Urtizberea, A.; Cremades, E.; Alonso, P.J.; Camón, A.; Ruiz, E.; Luis, F. Origin of slow magnetic relaxation in Kramers ions with non-uniaxial anisotropy. Nat. Commun. 2014, 5. [CrossRef] [PubMed]

25. Pedersen, K.S.; Dreiser, J.; Weihe, H.; Sibille, R.; Johannesen, H.V.; Sørensen, M.A.; Nielsen, B.E.; Sigrist, M.; Mutka, H.; Rols, S.; et al. Design of single-molecule magnets: Insufficiency of the anisotropy barrier as the sole criterion. Inorg. Chem. 2015, 54, 7600-7606. [CrossRef] [PubMed]

26. Bailey, A.; Griffith, W.P.; Leung, D.W.C.; White, A.J.P.; Williams, D.J. Ozonolysis for the preparation of high-oxidation state transition metal complexes, and the X-ray crystal structure of $\mathrm{Co}(\mathrm{py})_{2}(\mathrm{OAc})_{2}\left(\mathrm{H}_{2} \mathrm{O}\right)_{2}$. Polyhedron 2004, 23, 2631-2636. [CrossRef]

27. Aquilante, F.; Autschbach, J.; Carlson, R.K.; Chibotaru, L.F.; Delcey, M.G.; de Vico, L.; Fdez Galván, I.; Ferré, N.; Frutos, L.M.; Gagliardi, L.; et al. MOLCAS 8: New capabilities for multiconfigurational quantum chemical calculations across the periodic table. Comp. Chem. 2016, 37, 506-541. [CrossRef] [PubMed]

28. Figgis, B.N.; Hitchman, M.A. Ligand Field Theory and Its Applications, 1st ed.; Wiley-VCH: New York, NY, USA, 2000. 
29. Lloret, F.; Julve, M.; Cano, J.; Ruiz-García, R.; Pardo, E. Magnetic properties of six-coordinated high-spin cobalt(II) complexes: Theoretical background and its application. Inorg. Chim. Acta 2008, 361, 3432-3445. [CrossRef]

30. Chilton, N.F.; Anderson, R.P.; Turner, L.D.; Soncini, A.; Murray, K.S. PHI: A powerful new program for the analysis of anisotropic monomeric and exchange-coupled polynuclear d- and f-block complexes. J. Comp. Chem. 2013, 34, 1164-1175. [CrossRef] [PubMed]

31. Abragam, A.; Bleaney, B. Electron Paramagnetic Resonance of Transition Ions; Oxford University Press: Oxford, UK, 1970.

32. Pointillart, F.; Golhen, S.; le Guennic, B.; Guizouarn, T.; Ouahab, L.; Cador, O. Magnetic memory in an isotopically enriched and magnetically isolated mononuclear dysprosium complex. Angew. Chem. Int. Ed. Engl. 2015, 54, 1504-1507. [CrossRef] [PubMed]

33. Roos, B.O.; Lindh, R.; Malmqvist, P.-Å.; Veryazov, V.; Widmark, P.O. Main group atoms and dimers studied with a new relativistic ANO basis set. J. Phys. Chem. A 2004, 108, 2851-2858. [CrossRef]

34. Roos, B.O.; Lindh, R.; Malmqvist, P.-Å.; Veryazov, V.; Widmark, P.O. New relativistic ANO basis sets for transition metal atoms. J. Phys. Chem. A 2005, 109, 6575-6579. [CrossRef] [PubMed]

(C) 2016 by the authors; licensee MDPI, Basel, Switzerland. This article is an open access article distributed under the terms and conditions of the Creative Commons Attribution (CC-BY) license (http://creativecommons.org/licenses/by/4.0/). 